\title{
Methane emissions from landfills and carbon dynamics of harvested wood products: the first-order decay revisited
}

Kim Pingoud (kim.pingoud@metla.fi)

Fabian Wagner (fabian@iiasa.ac.at)

\section{Approved by}

Markus Amann(amann@iiasa.ac.at)

Leader, Atmospheric Pollution and Economic Development Program

February 2006 


\begin{abstract}
The First-Order decay method for carbon stocks is derived from first principles, and applications in the context of national greenhouse gas inventories are discussed. For methane emissions from landfills a method is developed that in principle is more accurate than the method currently recommended by the IPCC, and systematic errors are estimated numerically. The First-Order decay method is further applied to derive the permanent part of the carbon pool of harvested wood products.
\end{abstract}




\section{Acknowledgments}

We would like to thank Per Svardal and Riitta Pipatti for useful discussions and the two anonymous reviewers for constructive comments. 


\section{Contents}

\section{Contents}

1 Introduction $\quad 1$

2 The First Order Decay method for carbon stocks 4

2.1 The C-stock equation . . . . . . . . . . . . . . . . 4

2.2 Simple inflow models . . . . . . . . . . . . . . . . . . . 4

2.2.1 Continuous constant inflow . . . . . . . . . . . 5

2.2 .2 Instantaneous inflow . . . . . . . . . . . . . . 5

2.3 The C-stock equation with a delay constant . . . . . . . . . . . . 6

3 Applications $\quad \mathbf{8}$

3.1 Methane emissions from solid waste disposal sites . . . . . . . . . . . . . . 9

3.2 Harvested wood products . . . . . . . . . . . . . . 10

4 Numerical results $\quad 12$

5 Conclusion $\quad 13$ 


\title{
Methane emissions from landfills and carbon dynamics of harvested wood products: the first-order decay revisited
}

\author{
Kim Pingoud (kim.pingoud@metla.fi) \\ Fabian Wagner (fabian@iiasa.ac.at)
}

\section{Introduction}

Anthropogenic emissions of greenhouse gases are considered a significant factor in recent and future changes in the atmosphere's radiative budget and hence the global climate. In order to devise strategies for reducing these emissions it is necessary to determine the type and magnitude of the sources and to estimate the potentials for mitigation. An essential element of the United Nations Framework Convention on Climate Change (UNFCCC) is the National Greenhouse Gas Inventories that countries are committed to prepare on a regular basis. These inventories are prepared at a subsectoral level of disaggregation that allows the application of a wide range of methodologies for major greenhouse gases. The Intergovernmental Panel on Climate Change (IPCC) has provided internationally agreed methodologies [IPCC, 1997, IPCC, 2000, IPCC, 2003] that countries use to estimate their greenhouse gas emissions, and efforts are currently under way to update and strengthen the methodologies to reflect recent progress in scientific understanding and experience in inventory preparation.

It appears that one of the most challenging parts of the inventory methodology is estimating and reporting of biomass carbon pools. This is due to their dynamics: to estimate present emissions it is necessary to trace activities and relevant factors, such as those representing management practices, over long periods of time. Also, often the processes underlying the dynamics are highly uncertain, or the dynamics can only be approximated. Examples of such carbon pools are for instance forest biomass pools and harvested wood products (HWP) in the Agriculture, Forestry and Other Land Use sector and solid waste disposal sites (SWDS) in the Waste sector. In this context, but also in general, it is of vital importance to devise methods that make optimal use of the data generally available, and neither over- nor underestimate emissions and removals, as far as can be judged. This is a key ingredient to what the IPCC has termed good practice in inventory preparation.

This paper presents a general method that in principle could be used in national greenhouse gas inventories to describe the decay process of some biomass carbon stocks. The basic assumption is that the true dynamic process can be approximated by a first order decay (FOD) leading to an exponential decay pattern. Thus we will take for granted the assumptions made in the existing IPCC methodology [IPCC, 1997, IPCC, 2000, IPCC, 2003]

\footnotetext{
*Finnish Forest Research Institute, Unioninkatu 40 A, FI-00170 Helsinki, Finland and Technical Research Centre of Finland (VTT), P.O. Box 1602 , FI-02044 VTT, Espoo, Finland
} 
on the applicability of the FOD models. Our objective is rather to present a mathematically more exact and elegant solution under the above assumptions than to question the assumptions themselves. To illustrate the method it is specifically applied to describe methane emissions from SWDS and carbon balance of HWP in use and in SWDS. The method takes into account that both emission estimates in national inventories as well as the input data for the calculations are typically available on an annual basis.

Marland and Marland (2003) consider modeling the dynamics of carbon-containing long-lived products in the context of National Greenhouse Gas Inventories. They present solutions and their asymptotic behavior for some special cases of FOD, including constant production (=constant inflow to the product pool), exponentially growing, linearly growing and linearly declining production. The asymptotic solutions are very useful and characterize the behavior of the carbon stocks in case when there are no good statistics of the production or inflow rates, only some qualitative assumptions on their trends. In this paper we focus on cases where national activity data from the past are available, including for instance yearly HWP production, imports and exports rates or organic waste disposal rates into SWDS.

FOD is one of the simplest ways to describe dynamic processes. However, it can be noted that the decay pattern of long-lived product pools - or biomass pools in SWDS - is generally somewhat different: unlike FOD, the real decay pattern appears to be distributed so that the decay is small in the beginning of the process and the maximum decay takes place in later time instant, but the tail of the decay curve resembles FOD pattern. Row and Phelps (1996) proposed the use of a three-segment curve for HWP. Marland and Marland (2003) notice that the gamma distribution could be closely fitted to above curve and that it would provide an elegant mathematical option of describing the real process.

In HWP models also other decay patterns have been used. Karjalainen et al. (1994) use in their HWP model a logistic curve which resembles exponential decay. Ford-Robertson (2003) has also considered linear decay and compared it with exponential decay.

The main weakness of the FOD model is that it does not make any distinction between different elements within the pool of HWP in use, the decay only depending on the quantity of the total pool. In reality the age distribution of the HWP pool is decisive, which together with the estimated lifetime distribution of HWP determines the decay. For instance, it is obvious that the decay from old age classes of a certain wood product is higher than from young age classes. This kind of age distribution models would increase the mathematical complexity of the problem, but could as well be adopted to the IPCC methodology in the future.

The anaerobic decay of degradable organic carbon (DOC) in SWDS appears to be a process starting after a latent time period which could be modeled using delay (IPCC 1997, Bergman 1995, Barlaz 2004, Fischer et al. 2001). Combination of delay and FOD in the mathematical model might be the simplest way to approximate this decay process in SWDS and also acceptable for the purposes of national greenhouse gas reporting. Then one additional time parameter, the delay time, would be required. Besides, a portion of DOC appears to decay extremely slowly in anaerobic conditions, or even forming a permanent carbon stock in SWDS having a simple mathematical description.

Instead of the above FOD models more physically and process-oriented models could in principle be developed for the decay processes in SWDS. The generic paper of modeling aerobic decay (Hyvönen et al. 1998) has addressed the issue of estimating long-term effects of decay through short-term information. The major message of this paper is that simple models based on extrapolation from short-term observations may lead to errors in steadystate organic matter stores of a factor of 3 or more. Analogously to above, the use of 
unsophisticated FOD models could involve similar uncertainties in predicting long-term carbon balance in SWDS.

More accurate description of the decay processes can also be accomplished with FOD or other models by using several pools, representing products with various lifetimes or different processes associated with oxidization of the product pools. For example, Karjalainen et al.(1994) divide in their HWP carbon model the production flow of semi-finished products (e.g. sawn wood, plywood, particle board) into different shares placed to long and medium lifetime pools and to short-term use, reflecting in this model the existing wood use practice in Finland. This further increases the amount of model parameters to be estimated.

A specific problem in modeling dynamics of product pools - such as HWP in use is that their lifetime parameters are usually not physical or chemical constants, but also defined by socio-economic factors like trade cycles. Consequently, half-life of products in use is not time-invariant and it is difficult to provide any theoretically based and generally applicable values for product lifetimes in above dynamic models regardless of the decay pattern applied. One outcome of this difficulty would be direct inventory of carbon stocks, for example, estimation of amount of wood materials in specific end-uses like buildings in different time instances (see e.g. Pingoud et al. 1996 and 2001, Flugsrud et al. 1996). In this case no assumptions of product lifetimes are needed. .asically this technique is applicable only in some few countries due to lacking statistics on carbon-containing product stocks. (Information on production rates is often available, but not on the corresponding stocks.) However, this kind of inventories could potentially be used to estimate lifetime parameters which could be used in dynamic models more generally.

The objective of the IPCC Guidelines is to provide so-called tiered methodologies to estimate national carbon balance, for instance, in the dynamic carbon pools. At Tier 1 no country-specific methods are used, only activity data of the given country. Most parameter values are defaults provided by the Guidelines. Higher tiers may use the same estimation models but including additional country-specific data: carbon conversion factors, half-life of products, number of product or waste pools could be different. At the highest tier level 3 also different and complementary methods (such as inventories) can be used and the methods could be based on elaborated analysis of all the associated HWP carbon flows within the country (e.g. Japan, see Hashimoto and Moriguchi 2004).

The objective of this paper is to provide simple methods based on analytical solution of FOD differential equation that could be applied globally in national inventories at the lowest tier levels, being a slight improvement on the existing methods. For comparison, in the 2003 Good Practice Guidance of IPCC the suggested Tier 1 and Tier 2 methods for HWP are based on numerical finite-difference approximation of the FOD equations. In the 2000 Good Practice Guidance of IPCC the presented solution for FOD in SWDS is clumsy and mathematically inexact. The basic statistics on HWP to be applied is the global FAO statistics providing yearly national data since 1961 . These statistics enable estimation of carbon dynamics of semi-finished HWP pools by FOD models considered in this paper. The final products are implicitly included in above pools and immediate losses of the pools due to final processing (e.g. in construction) are assumed to be included and described realistically by the exponential decay pattern. For the estimation of the carbon balance in SWDS no statistics are collected at the global level and data availability among countries varies. For SWDS the delayed FOD decay model together with a model for permanent carbon sequestration into SWDS are assumed to describe realistically the behavior of degradable organic carbon in SWDS. The structure of this paper is as follows. First we derive a formula for the total carbon stock at the end of each inventory year. This 
is done for two alternative models representing the inflow of carbon into the carbon pool. We then derive analogous results under the assumption that the dynamic decay is delayed (e.g., in SWDS by the acidification process). We then apply these general results to SWDS and HWP and give some numerical examples, before we end with some conclusions.

A version of the FOD method has already been used by the IPCC in the context of SWDS. In this paper we derive this IPCC version as a special case in our general setup, but show it leads to a systematic and significant underestimation of emissions. Furthermore, we propose a more realistic FOD method that could supersede the method currently used by the IPCC.

\section{The First Order Decay method for carbon stocks}

In this section we describe the dynamics of carbon stocks that are subject to a FOD. However, in practice not all of the stocks in a given pool may be subject to such decay, or the decay may be delayed. It is therefore necessary to clearly distinguish the decaying carbon stock at time $t$, which we denote by $S(t)$, from the inert part of the stock, which we denote by $\widehat{S}$. The total carbon stock at any time is then the sum of the decaying part and the inert part

$$
S_{\text {Total }}(t)=S(t)+\widehat{S}(t)
$$

Let us now first discuss the dynamics of the decaying part.

\subsection{The C-stock equation}

The stock change $\frac{d S}{d t}(t)$ at time $t$ has two components: the inflow $I(t)$ into the stock, and the outflow $O(t)$, which depends on the stock at time $t$ :

$$
\frac{d S}{d t}(t)=I(t)-O(t)=I(t)-k \cdot S(t) .
$$

Here $k$ is the decay constant, and for zero inflow the solution of Eq.(2) is given by the simple exponential decay:

$$
S(t)=S_{0} \cdot e^{-k\left(t-t_{0}\right)}, \quad S\left(t_{0}\right) \equiv S_{0}
$$

with a half-life of $t_{1 / 2}=\frac{\ln 2}{k}$. For non-zero inflow $I(t)$, however, the general solution of Eq.(2) is given by

$$
S(t)=\left(\int_{t_{0}}^{t} I\left(t^{\prime}\right) \cdot e^{-k\left(t-t^{\prime}\right)} d t^{\prime}\right)+S_{0} \cdot e^{-k\left(t-t_{0}\right)}
$$

The first term describes the contribution of the inflowing carbon to the current stock at time $t$, whereas the second term describes the remainder at time $t$ of the initial stock at time $t_{0}$. For general $I(t)$ the integral in Eq. (4) can be solved numerically; in the following we discuss important special cases in which it can also be solved analytically.

\subsection{Simple inflow models}

The carbon inflow function $I(t)$ is a function that represents the actual amount of carbon that flows into the carbon stock over time. The inflow may depend on a number of parameters and its exact form may be difficult to determine, in particular at the national level. It is therefore useful to take a pragmatic view and use simplified approximations to the actual inflow function. Two of such approximations are presented here; both take into account that, at the national level, inflow data are typically available on an annual basis. The two approximations differ in the representation of the timing of the inflow. 


\subsubsection{Continuous constant inflow}

For the continuous constant inflow model it is assumed that the inflow rate does not change over time and its value is determined by dividing the total amount of inflow by the time period during which the inflow occurred. In this case Eq. (4) simplifies to

$$
S_{\text {cont. }}(t)=\frac{I}{k} \cdot\left(1-e^{-k\left(t-t_{0}\right)}\right)+S_{0} \cdot e^{-k\left(t-t_{0}\right)}
$$

If the inflow rate is constant only within an interval, say one year, but changes over the years $\left(i . e . I(t)=I_{i}\right.$ for $T_{i} \leq t<T_{i+1}, i=0, \ldots, n$, where $T_{i}$ denotes the beginning of year $i)$, then Eq. (4) can be written for $t=T_{n+1}$, i.e. the end of year $n$ :

$$
S_{\text {cont. }}\left(T_{n+1}\right)=\sum_{i=0}^{n} I_{i} \cdot \frac{e^{k}-1}{k} \cdot e^{-k\left(T_{n}-T_{i}\right)}+S_{0} \cdot e^{-k\left(T_{n+1}-T_{0}\right)}
$$

where we have performed the integration over exponentials and used the fact that $T_{i+1}=$ $T_{i}+1$. Eq.(6) is rather useful in the context of annual stock or emission inventories because typically the total inflow in each year is can be estimated, even though the actual inflow rate at each point in time is not known. For example, one may estimate from municipal waste statistics the amount of DOC that has been deposited into a landfill during a given year without knowing how many trucks brought in how much DOC on each day of the year. Dividing the total inflow by the inflow period (here: one year) results in the average inflow rate for one year. The same applies to statistics of harvested wood products: only yearly production rates are compiled. Thus it is reasonable to assume that the production and input flow into the HWP pool is evenly distributed over the year.

The above Eq. (6) can also be presented in recursive form in which the stock at the beginning of year $n+1$ can be expressed as a function of stock at the beginning of year $n$ and the constant inflow during year $n$ :

$$
S_{\text {cont. }}\left(T_{n+1}\right)=e^{-k} \cdot S_{\text {cont. }}\left(T_{n}\right)+\frac{1-e^{-k}}{k} \cdot I_{n}
$$

This equation is especially practicable as it gives a simple updating rule for subsequent inventories.

\subsubsection{Instantaneous inflow}

In contrast to the constant inflow model above, for the instantaneous inflow model it is assumed that the total inflow occurs at a single point in time. For instance, if only the total amount of C-inflow into the carbon stock of a SWDS is known only per year, but not at higher resolution in time, it will be assumed that all the DOC was deposited, say, on 1 January of that year, or on 1 July, or some other date.

Mathematically the instantaneous inflow is represented by a so-called delta-function $\delta(x)$ which has the property

$$
\delta(x)=0 \quad \text { except for } x=0, \quad \text { and } \int f(x) \delta\left(x-x_{0}\right) d x=f\left(x_{0}\right)
$$

if $x_{0}$ lies in the integration domain. The delta function essentially peels off the integral and leaves the integrand with the appropriate argument. In order to avoid double counting the integration domain needs to be defined carefully. For example, here the convention is used that $T_{i}$ belongs to the year $i$ ( $T_{i}$ being 1 January of that year), and consequently 
$T_{i+1}$ does not (being 1 January of the following year). In particular it follows that for the integral over year $i$

$$
\int_{T_{i}}^{T_{i+1}} \delta\left(t^{\prime}-T_{i+1}\right) d t^{\prime}=0
$$

because $T_{i+1}$ does not lie inside the integration domain.

If the instantaneous inflow $I$ occurs at $t=t^{*} \geq T_{0}$, one has $I(t)=I \cdot \delta\left(t-t^{*}\right)$, and Eq.(4) becomes

$$
S_{\text {inst. }}\left(T_{n+1}\right)=I \cdot e^{-k\left(T_{n+1}-t^{*}\right)}+S_{0} \cdot e^{-k\left(T_{n+1}-T_{0}\right)}
$$

i.e. $S_{0}$ is decaying since $T_{0}$ and $I$ is decaying only since the time when the inflow occurred.

In the compilation of an annual inventory it is necessary for consistency to assume that each year the instantaneous inflow of $I_{i}$ occurs on the same date. If $a$ denotes the inflow date (in units of years, i.e. $0 \leq a<1$ ), then the inflow in year $i$ can be expressed as

$$
I(t)=I_{i} \cdot \delta\left(t-\left(T_{i}+a\right)\right), \quad T_{i} \leq t<T_{i+1}
$$

Hence the annualized version of Eq. (4) becomes

$$
S_{\text {inst. }}\left(T_{n+1}\right)=\sum_{i=0}^{n} I_{i} \cdot e^{-k\left(T_{n+1}-T_{i}-a\right)}+S_{0} \cdot e^{-k\left(T_{n+1}-T_{0}\right)}
$$

and describes the stock at time the end of the inventory year $\left(t=T_{n+1}\right)$ assuming an instantaneous inflow of carbon each year on a date $a$ (the value $a=0$ corresponds to 1 January, $a=\frac{1}{2}$ to 1 July, etc.).

Again, the stock at the end of each year can be expressed as a function of the stock at the beginning of the year (end of the previous year) and the total inflow into the stock

$$
S_{\text {inst. }}\left(T_{n+1}\right)=e^{-k} \cdot S_{\text {inst. }}\left(T_{n}\right)+I_{n} \cdot e^{-k(1-a)}
$$

It can easily be seen that Eq.(6) and Eq.(12) (and similarly their recursive analogues Eq.(7) and Eq.(13)) coincide if and only if

$$
a=a^{*}=\frac{1}{k} \ln \left(\frac{e^{k}-1}{k}\right)
$$

Thus one can always choose the inflow date $a$ such that the instantaneous inflow model results reproduce the results derived with the more realistic continuous inflow model. However, $a^{*}$ depends on $k$, so if the carbon stock has components with distinct decay constants $k$, different values for $a^{*}$ have to be chosen for these components. Figure 1 illustrates the dependency of $a^{*}$ on $t_{1 / 2}$. It can be seen that $a^{*} \approx 1 / 2$, so that the instantaneous inflow model with $a=1 / 2$ ( 1 July) is a fairly good approximation for a half life of more than 1 year $\left(t_{1 / 2}>1\right)$. For a half life less than half a year the instantaneous inflow model with $a=1 / 2$ is no longer a good approximation.

\subsection{The C-stock equation with a delay constant}

In the previous sections we have considered the dynamics of the decaying part of the stocks. The inflow of carbon was assumed to be part of the decaying component of the stock, while the inert part of the stock remained unchanged. In the context, e.g. of SWDS, however, it is useful to consider also the case where the inflowing carbon remains 


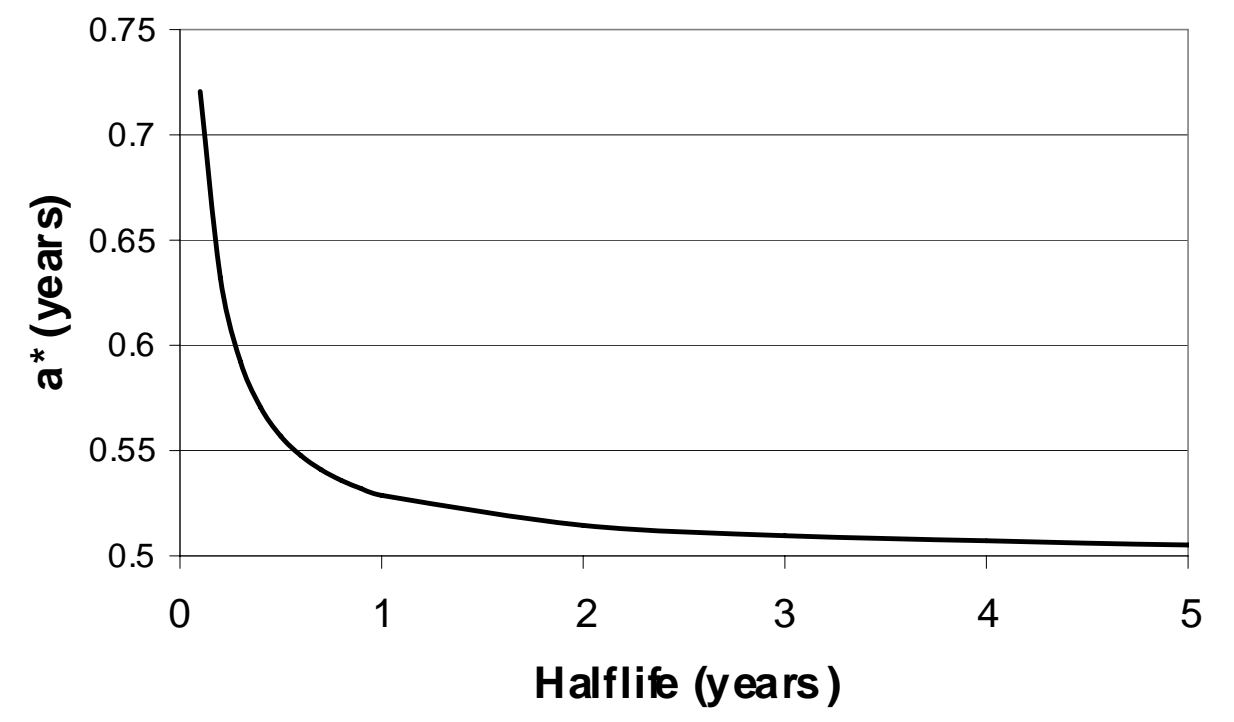

Figure 1: The value of $a=a^{*}$ for which the results of the instantaneous and the continuous inflow model agree, as a function of the half-life of the decay.

inert for some time before acidification has reached a level at which an anaerobic decay commences. Such a delay in the decay process can be represented by a delay constant $\Delta$ (here we consider $0 \leq \Delta<1$ in units of years) in Eq. (2):

$$
\frac{d S}{d t}(t)=I(t-\Delta)-k \cdot S(t)
$$

The inflow into the total stock at an earlier time $t-\Delta, I(t-\Delta)$, determines the inflow into the decaying stock at time $t$. The inert part of the stock changes according to

$$
\frac{d \widehat{S}}{d t}(t)=I(t)-I(t-\Delta) .
$$

With this the continuous inflow model Eq.(6) for the decaying part of the stock then is replaced by

$$
\begin{gathered}
S_{\text {cont. }}\left(T_{n+1}\right)=\sum_{i=0}^{n-1} I_{i} \cdot \frac{e^{k}-1}{k} \cdot e^{-k\left(T_{n+1}-T_{i}-\Delta\right)}+I_{n} \cdot \frac{1-e^{-k(1-\Delta)}}{k} \\
+S_{0} \cdot e^{-k\left(T_{n+1}-T_{0}\right)}
\end{gathered}
$$


and we have $\widehat{S}\left(T_{i+1}\right)=\Delta \cdot I_{i}$ for the inert part of the stock. With this it is not difficult to derive a recursive formula for the total stock at the end of year $n$ :

$$
\begin{array}{r}
S_{\text {Total, cont. }}\left(T_{n+1}\right)=S_{\text {cont. }}\left(T_{n+1}\right)+\widehat{S}_{\text {cont. }}\left(T_{n+1}\right) \\
=e^{-k} \cdot S_{\text {Total, cont. }}\left(T_{n}\right)+I_{n} \cdot\left(\frac{1-e^{-k(1-\Delta)}}{k}+\Delta\right) \\
+I_{n-1} \cdot e^{-k} \cdot\left(\frac{e^{k \Delta}-1}{k}-\Delta\right)
\end{array}
$$

Note that the $I_{n-1}$-term is zero for $\Delta=0$ so that, in this case, we recover Eq.(7) above.

The analysis of the delay in the inflow can now easily be extended to the instantaneous inflow model in which the parameter $a$ represents a single deposition date. With the delay the decaying carbon stock at the end of the inventory year $n$ Eq.(12) is replaced by

$$
S\left(T_{n+1}\right)_{\text {inst. }}=\sum_{i=0}^{n} I_{i} \cdot e^{-k\left(T_{n+1}-T_{i}-a-\Delta\right)}+S_{0} \cdot e^{-k\left(T_{n+1}-T_{0}\right)}
$$

In deriving the corresponding recursive formula one has to distinguish the cases $a+\Delta<1$ and $a+\Delta \geq 1$. If $a+\Delta<1$ the carbon that went into the total stock during year $i$ $\left(I_{i}\right.$ at date $\left.a\right)$ is already assumed to be decaying at the end of year $i$. Hence in this case $\widehat{S}\left(T_{i}\right)=0$. On the other hand, if $a+\Delta \geq 1$, then all of $I_{i}$ is still inert at the end of year $i$, hence $\widehat{S}\left(T_{i}\right)=I_{i}$. Thus one finds

- for $a+\Delta<1$ :

$$
\begin{aligned}
& S_{\text {Total, inst. }}\left(T_{n+1}\right) \\
& \quad=e^{-k} \cdot S_{\text {Total, inst. }}\left(T_{n}\right)+I_{n} \cdot e^{-k(1-a-\Delta)}
\end{aligned}
$$

- $a+\Delta \geq 1$ :

$$
\begin{aligned}
& S_{\text {Total, inst. }}\left(T_{n+1}\right) \\
& \quad=e^{-k} \cdot S_{\text {Total, inst. }}\left(T_{n}\right)+I_{n} \cdot\left(1+e^{-k(1-a-\Delta)}\right)-e^{-k} \cdot I_{n-1}
\end{aligned}
$$

It is clear that the instantaneous deposition model with parameters $a$ and $\Delta$ is equivalent to an instantaneous deposition model with a deposition date $a^{\prime}=a+\Delta$ and no delay $\left(\Delta^{\prime}=0\right), c f$. Eqs. (19) and (12). Thus, a delay in the decay process can be modeled as a delay in the deposition.

However, when we compare the continuous and the instantaneous deposition models with delay we note that, in contrast to the model without a delay constant $\Delta$, an analytic expression for $a=a^{*}$ in terms of $k$ and $\Delta$, for which Eq.(17) and Eq.(19) coincide, cannot be given. Whether the instantaneous inflow model is a good approximation to the more realistic continuous inflow model, and for which value of $a$, can only be decided numerically, and we will give some examples in Section 4.

\section{Applications}

In this section we apply the results obtained in the previous section to specific circumstances relevant in the context of annual greenhouse gas inventories. 


\subsection{Methane emissions from solid waste disposal sites}

Landfill gas, consisting of roughly half methane and half carbon dioxide (on volume basis), is generated through anaerobic decay (methanogenic bacteria) from degradable organic carbon (DOC) deposited in landfills. The generation of methane appears not to start immediately after deposition. The gas can migrate from SWDS laterally or by venting to the atmosphere. Some part of the methane generated in the anaerobic part of the landfill is oxidized to carbon dioxide in the upper aerobic part through bacteria processes. In fact only one portion, $\mathrm{DOC}_{f}$, of the DOC inflow is actually decaying in anaerobic conditions, the rest of the DOC inflow, $\left(1-\mathrm{DOC}_{f}(t)\right) \cdot \mathrm{DOC}(t)$, appears to form a nearly permanent carbon stock. The factor $\mathrm{DOC}_{f}$ is dependent on the types of DOC deposited and types SWDS, which may differ from each other within a country.

A number of factors, such as waste disposal practices and physical parameters determine the rate at which the methane is generated from the decaying DOC. The IPCC [IPCC, 1997] has documented the FOD method as a viable route for estimating annual methane emissions. Here we apply the above results from the FOD to SWDS, including the different deposition models and the delay factor, and comment briefly on the relation of our findings to the IPCC methodology. Only the anaerobic decay process is considered in the following, not the aerobic decomposition typical in open dumps.

\section{Carbon stocks and methane emissions}

As only methane emissions from SWDS are reported in the national GHG inventories but not the permanent sequestration of DOC into SWDS - solely the dynamics of the decaying DOC need to be considered in the estimation method. The dynamics can be described by the delayed FOD model presented in Eqs. (15)-(18). When applying the FOD method it is realistic to use a multiple-carbon-pool model (comprising typically 2-4 separate pools) with different decay constants for various waste components or different SWDS types. The inflow, $\mathrm{DOC}_{f}$. DOC(t), is diverted into these 2-4 pools based on waste composition and the type of SWDS.

Continuity requires that the methane generated during year $n$ is proportional to the net outflow of carbon, which is the total stock change minus the total inflow $I_{n}$ during year $n$ :

$$
\begin{aligned}
& \left.\mathrm{CH}_{4} \text { (generated during year } n\right) \\
& \quad=\lambda \cdot\left(S_{\text {Total }}\left(T_{n+1}\right)-S_{\text {Total }}\left(T_{n}\right)-I_{n}\right)
\end{aligned}
$$

where $\lambda$ is a conversion factor from mass of DOC to mass of methane, which includes the methane correction factor (MCF) and other parameters (for details see, e.g. [IPCC, 1997, IPCC, 2000]). In order to obtain an expression for the actual emissions one has to subtract the amount of methane that is recovered from the total methane generated.

Let us first look at the continuous inflow model. With the help of Eq.(18) it is easy to derive the methane generated during year $n$ as a function of the total stock at the beginning of year $n$ and the total inflow in years $n$ and $n-1$ :

$$
\begin{aligned}
& \left.\mathrm{CH}_{4} \text { (generated during year } n\right) \text { cont. } \\
& \quad=\lambda \cdot\left(\alpha_{k} \cdot S_{\text {Total,cont. }}\left(T_{n}\right)+\beta_{k, \Delta} \cdot I_{n}+\gamma_{k, \Delta} \cdot I_{n-1}\right)
\end{aligned}
$$

where the coefficients depend on the decay constant $k$ and the delay $0 \leq \Delta<1$ : 


$$
\begin{aligned}
\alpha_{k} & =e^{-k}-1 \\
\beta_{k, \Delta} & =\frac{1-e^{-k(1-\Delta)}}{k}+\Delta-1 \\
\gamma_{k, \Delta} & =e^{-k}\left(\frac{e^{k \Delta}-1}{k}-\Delta\right)
\end{aligned}
$$

For the instantaneous inflow model we use Eqs.(20) and (21) together with Eq.(22) to find an expressions for the methane generated during year $n$ as a function of the total stock at the beginning of year $n$ and the total inflow in years $n$ and $n-1$ :

- for $a+\Delta<1$

$$
\begin{aligned}
& \left.\mathrm{CH}_{4} \text { (generated during year } n\right)_{\text {inst. }} \\
& \quad=\lambda \cdot\left(\alpha_{k}^{\prime} \cdot S_{\text {Total, inst. }}\left(T_{n}\right)+\left(\beta_{k, a, \Delta}^{\prime}-1\right) \cdot I_{n}\right)
\end{aligned}
$$

- for $a+\Delta \geq 1$

$$
\begin{aligned}
& \left.\mathrm{CH}_{4} \text { (generated during year } n\right)_{\text {inst. }} \\
& \quad=\lambda \cdot\left(\alpha_{k}^{\prime} \cdot S_{\text {Total, inst. }}\left(T_{n}\right)+\beta_{k, a, \Delta}^{\prime} \cdot I_{n}-\gamma_{k}^{\prime} \cdot I_{n-1}\right)
\end{aligned}
$$

where the coefficients are

$$
\begin{aligned}
\alpha_{k}^{\prime} & =e^{-k}-1=\alpha_{k} \\
\beta_{k, a, \Delta}^{\prime} & =e^{-k(1-a-\Delta)} \\
\gamma_{k}^{\prime} & =e^{-k}
\end{aligned}
$$

We observe that for $\Delta=0$ the results for the two inflow models coincide for $a=a^{*}$ as in Eq. (14), as they should from what we have observed for the corresponding carbon stocks above.

We further observe that the FOD method described in the 1996 IPCC Guidelines is implicitly using an instantaneous inflow model without delay and the inflow date 1 January, i.e. $a=0$ and $\Delta=0$. This choice for $a$ is inappropriate and introduces a substantial error in the estimation of the carbon stock and methane generated/emitted during the inventory year. For example, with a half life of 2 years, the error in the methane generation relative to the constant inflow model is 20 percent (underestimation), and for half life of 5 and 10 years, the errors are still 7 and 3 percent, respectively. Since these are systematic errors, they should be avoided and a more accurate method should be used, even in view of the large uncertainties in the understanding of the underlying processes. The continuous inflow model provides such a more accurate method, but a better choice for $a$ could lead to accurate results too (see Section 4).

\subsection{Harvested wood products}

Carbon balance in harvested wood products (HWP) is an issue that apparently will be included in the forthcoming 2006 IPCC Guidelines for national GHG inventories. Undecided is still the reporting approach, which determines how those emissions are allocated among countries in different position (wood producers, importers and exporters). However, regardless of the approach, carbon stock change in HWP pools is the basic quantity to be estimated in order to calculate the national $\mathrm{C}$ balance according to each approach. 
The simplest way to model the dynamics of HWP in use is the FOD process without delay discussed in this paper. However, the lifetime of HWP in different end-uses varies: there are very short-lived products such as wood fuels or advertising papers and long-lived ones such as construction wood, whose lifetime in service can be even hundreds of years. To obtain a more realistic description of the real process it thus it advisable to use a model with multiple-pools having different half-lives. For instance, solid wood products (sawn wood and wood-based panels) and paper products could be divided into separate pools in the model, or also many more than two separate pools could be used. The half-life of paper products pool(s) could be of the order of 1 year and half-life of wood-based panels and sawn wood pools of the order of a couple of decades. Some very short-lived products like wood fuels can even be assumed to decay immediately after harvesting.

The only activity data that can be used globally for the purpose of national inventories on HWP in use are the historical production, export and import rates of various HWP. FAO has collected annual data from all countries [FAO, 2005] - mainly since 1961 - based on national submissions. Consequently the quality of these data varies. The above activity data can be used to calculate the inflows to the HWP pools of the FOD model. The inflow to the HWP pools can be approximated to be constant and evenly distributed within each year, as the activity data is only on yearly basis. The historical inflows before 1961 are of less importance for the accuracy of model estimates at present time. For instance, an assumption of exponential growth in past HWP consumption correlating with past roundwood production could be applied when calculating the historical inflows of the model.

Under the above assumptions the carbon dynamics of a HWP pool can be described recursively by Eq.(7), which means that having the stock estimate for 1 January of year $n$ the corresponding estimate for the next year $n+1$ can be generated. Further, the stock change during year $n$ is simply given by

$$
\begin{aligned}
& S_{\text {cont. }}\left(T_{n+1}\right)-S_{\text {cont. }}\left(T_{n}\right) \\
& \quad=\frac{1-e^{-k}}{k} \cdot I_{n}+\left(e^{-k}-1\right) \cdot S_{\text {cont. }}\left(T_{n}\right)
\end{aligned}
$$

The average $\mathrm{C}$ outflow from the HWP pool during year $n$ is given by

$$
O_{\text {cont. }}\left(T_{n}\right)=\left(1-\frac{1-e^{-k}}{k}\right) \cdot I_{n}+\left(1-e^{-k}\right) \cdot S_{\text {cont. }}\left(T_{n}\right)
$$

If activity data are available, the above equations can be integrated - starting far from the past with a zero initial stock to obtain an estimate of the present stock change or outflow rate. Or alternatively, having a stock estimate for some more recent year, estimates for subsequent years until the present one can be generated recursively.

Another HWP pool to be estimated and reported in national inventories - in case local activity date on HWP waste disposal are be available - are HWP in SWDS decaying in anaerobic conditions. All methane emissions from SWDS are reported according to existing practice concentrated under the Waste sector in national GHG inventories, but in the forthcoming 2006 IPCC Guidelines the carbon balance of the pool of HWP in SWDS will apparently be reported separately together with HWP in use under the Agriculture, Forestry and Other Land Use (AFOLU) sector. There are basically three DOC fractions of HWP in SWDS which must be modeled separately: One fraction of DOC in HWP (lignin and partly also other wood fractions) will never decay in anaerobic conditions of SWDS. This fraction forms an almost permanent storage having no outflow. The other two fractions were already described in Section 2.3: 1) the inert part consists of DOC 


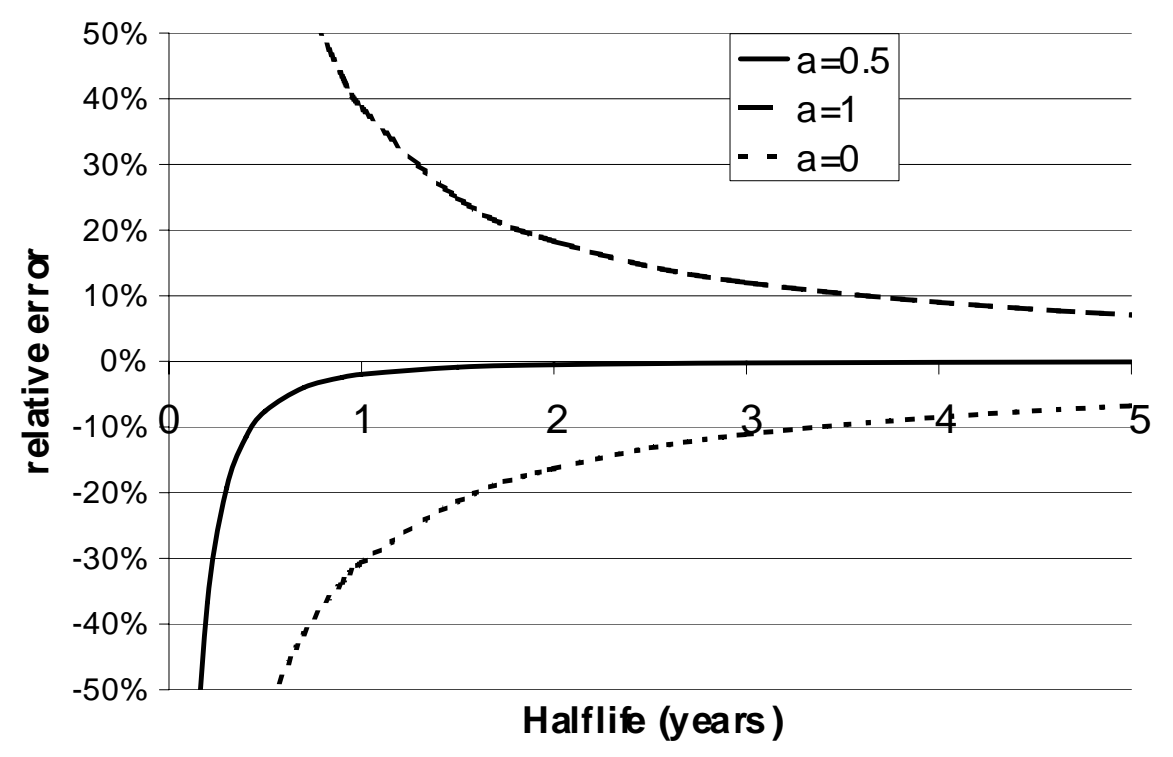

Figure 2: Error of the instantaneous model relative to the continuous model for different choices of inflow dates $a$ (in units of years), as a function of the half-life.

where the decomposition process has not yet started, and 2) the decaying part described by the delayed FOD model. This decaying part could in addition be divided into two or even more subpools with different half-lives dependent on the quality of wood-based waste (e.g. paper or solid wood). To estimate the C stock change of HWP in SWDS as a whole, stock changes in all above fractions must be summed up.

The dynamics of the permanent fraction can be described by the equation:

$$
\frac{d S_{\mathrm{perm}}(t)}{d t}=\left(1-\operatorname{DOC}_{f}(t)\right) \cdot \operatorname{DOC}(t), \quad S_{\text {perm }}\left(t_{0}\right)=S_{\text {perm }, 0}
$$

The solution for continuous constant inflow to permanent stock can be presented in the recursive form analogously to FOD (Eq. (7)):

$$
S_{\operatorname{perm}}\left(T_{n+1}\right)=\left(1-\mathrm{DOC}_{f, n}\right) \cdot \operatorname{DOC}_{n}+S_{\operatorname{perm}}\left(T_{n}\right)
$$

\section{Numerical results}

We now compare first the results of the carbon stock calculations for the continuous inflow model and the instantaneous inflow model in the case without delay $(\Delta=0)$. Figure 2 shows the relative error in the stock estimate that is made for various choices of the instantaneous inflow date $a$. For this case we have considered a stock that decays over forty years, and have assumed a zero initial stock.

One can observe that the model with inflow date 1 July $(a=1 / 2)$ gives fairly good results for the carbon stock for medium and long half-lives, the error relative to the continuous inflow model lies below 2 percent for half-lives longer than 1 year. However, 
for short half-lives, e.g. 5-6 months, the error is of the order of 10 percent and therefore significant, taking into account that it is systematic and can be avoided by using the continuous inflow model. Shorter half-lives are typically used when estimating changes of carbon stocks in HWP, especially paper products.

The graph also shows the corresponding relative errors in the carbon stocks calculated with the models with inflow dates at the beginning and the end of each year, respectively, among them the current IPCC method for estimating methane emissions from landfills $(a=0)$. It can be seen that both assumptions lead to systematic errors that, for short halflives, are larger than the overall uncertainty associated with the first-order decay method reported by UNFCCC Annex I parties. On the other hand, the large relative errors in short-lived carbon stocks do not necessarily lead to large absolute errors in estimates of the total carbon balance. In a multiple-pool model - by which the decay process will be described in practice - the carbon stocks with long half-lives are dominating the total balance, thus decreasing the absolute estimation error.

We have further analyzed how a non-zero delay constant $\Delta$ influences these findings by comparing the results obtained by Eq.(17) and Eq.(19). We had noted above that for the instantaneous inflow model a non-zero delay constant is equivalent to a delay in the inflow date, whereas in the continuous model the delay constant enters the expression for the carbon stock in different manner. Relative to the more realistic continuous model, the instantaneous model introduces another error into the carbon stock by treating the delay constant in this simplistic way.

Figure 3 illustrates this $\Delta$-induced error as a function of $\Delta$ and the half-life, and we focus on the case $a=1 / 2$, i.e. deposition occurs on 1 July in the instantaneous model. The bold graph is thus the same as the middle graph in Figure 2, and shows fairly small errors relative to the continuous model, at least for half-lives longer than 1 year. The other graphs show the corresponding errors for various values of $\Delta$, ranging from 0.1 to 0.5 years. The top graph thus corresponds to the instantaneous model in which the inflow occurs on 1 July, while the decay process only commences on 31 December of each year.

One can observe from Figure 3 that the longer the delay, the larger the error relative to the continuous model which uses the same delay constant $\Delta$ : as functions of the half-life $t_{1 / 2}$ the graphs converge slower for higher delay constants. One can now see that, if a delay occurs in the decay process $(\Delta>0)$, the choice $a=1 / 2$ will lead to substantial error in the stock calculation, especially for half-lives of 5 years or less, despite the fact that in the case without delay the choice $a=1 / 2$ lead only to small errors, at least for half-lives of 1 year or more (see above). In order to obtain better results, one would need to choose a different value for $a$, and this choice would depend on the half-life and the delay constant.

One can also observe as an aside that, though the $\Delta=1 / 2$ graph in Figure 3 looks very much like the $a=1$ graph in Figure 2, they are numerically different.

Finally, as in illustration, Figure 4 shows the total carbon stock and its permanent, inert and decaying parts over time, assuming no initial stock, a half life of 10 years, a 6 months delay in the decay process.

\section{Conclusion}

In this paper we have derived carbon stock equations from first principles, where we have used two different types of inflow model, the instantaneous inflow model and the continuous inflow model. We have presented a number of useful formulae for the carbon stock for the two models, in particular we have provided recursive formulae that can easily be used in 


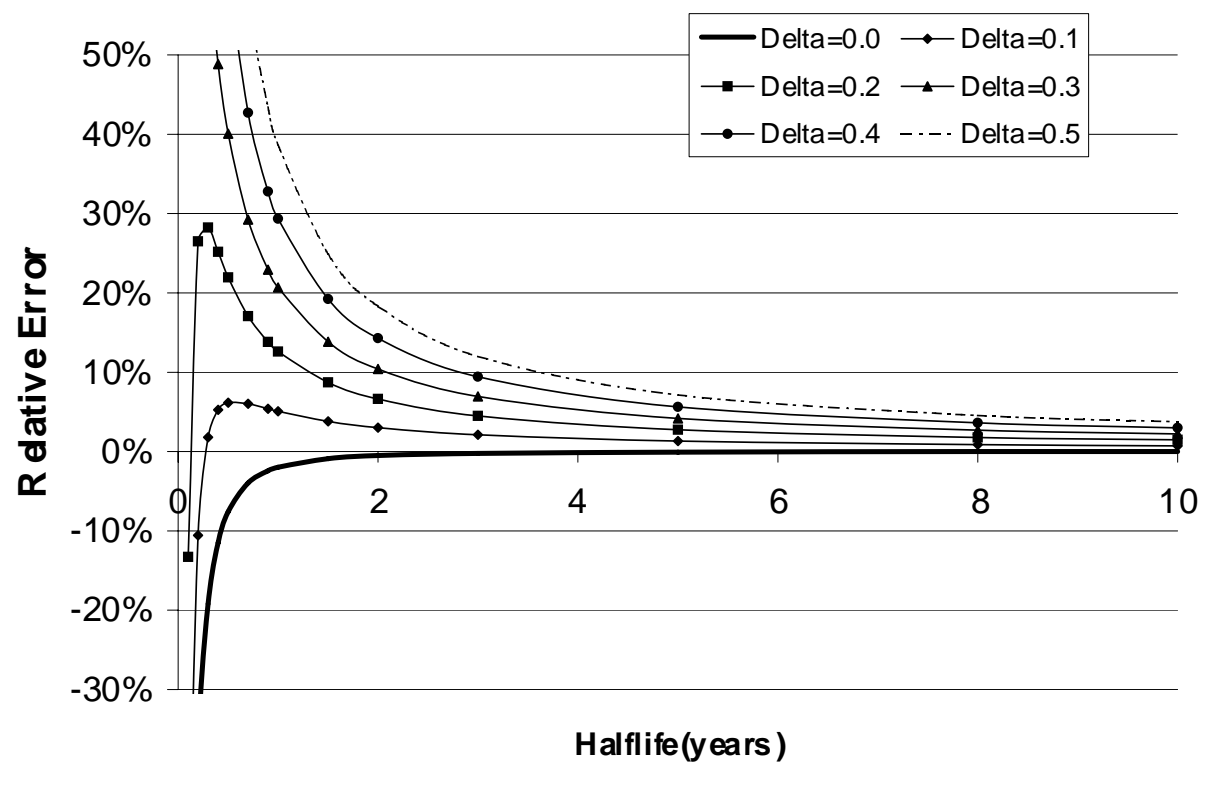

Figure 3: Error of the instantaneous model relative to the continuous model for the choice $a=\frac{1}{2}$ and various delays $\Delta$ (in units of years) in the decay, as a function of the half-life.

the context of annual national inventories since they only involve the stocks of the previous year and the most recent stock changes. This corresponds to the data typically available to the inventory compiler. All else being equal, the continuous inflow model is more realistic and is expected to provide more accurate results than the instantaneous inflow model.

We have further compared the results for the two inflow models numerically, both with and without an assumed delay in the decay process, and arrived at the following conclusions. For half-lives of more than 1 year and no delay, the instantaneous model provides a fairly accurate approximation to the more realistic continuous model. If a delay is assumed, it introduces substantial error into the instantaneous approximation, even for half-lives between 1 and 5 years. For short lifetimes, the error is large with or without delay.

The error can be corrected by an appropriate choice for the parameter $a$ in the instantaneous model. However, from a methodological point of view this is unsatisfactory, in particular in view of the fact that a method - the continuous model - exists which (1) is more realistic, (2) does not depend on the parameter value $a$, and (3) for which carbon stocks (and e.g. methane emission from landfills) can be calculated using formulas that are by no means more complex than those derived for the instantaneous model.

Analogous equations were derived for harvest wood products and their impact on carbon stocks assuming continuous inflow that remains constant over one year. Conclusions on error estimates can directly be translated into this context as well. More generally, as a relatively simple method the First-Order decay method paired with the continuous inflow model is as realistic as possible given the small number of parameters, and provides more accurate results than the methods in earlier IPCC publications. 


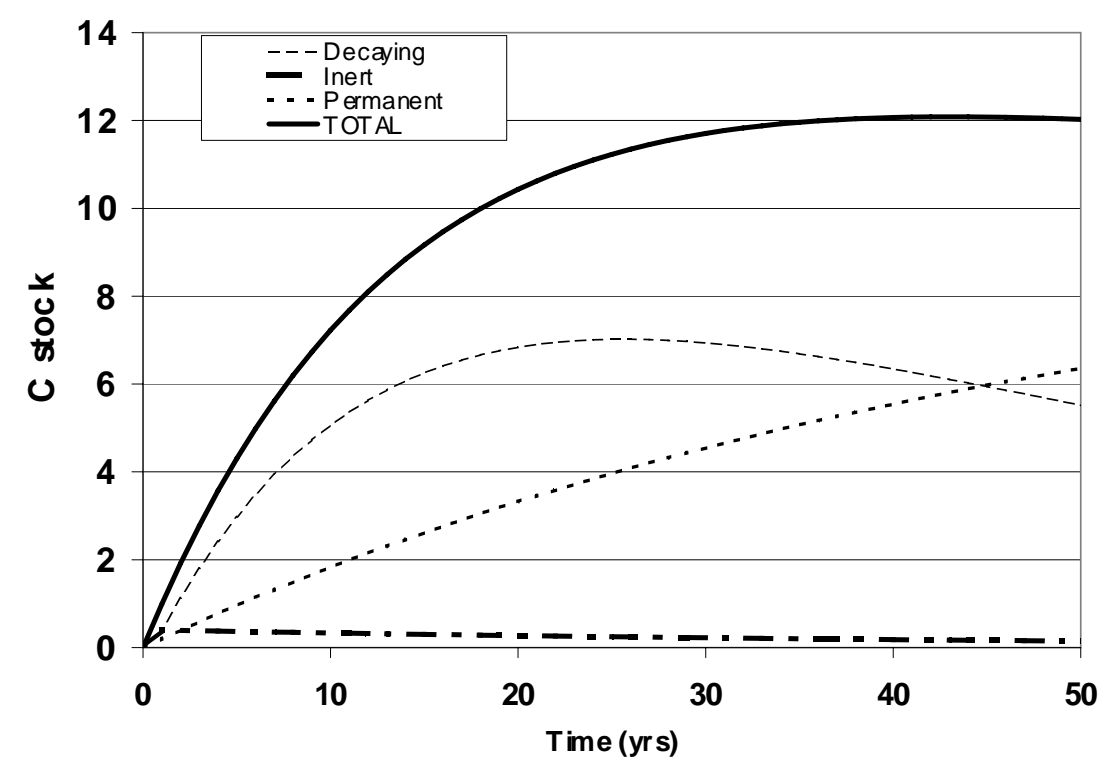

Figure 4: Example of the composition of the total carbon stock in solid waste disposal sites over time, assuming an initial annual inflow of 1 unit per year, decreasing by 2 percent per year (half-life 10 years, $\mathrm{DOC}_{f}=0.8, \Delta=0.5$ years and zero initial carbon stock)

\section{References}

[Barlaz, 2004] Barlaz, M. Critical review of forest products decomposition in municipal solid waste landfills. Technical Bulletin no. 872, Research Triangle Park, N.C.: National Council for Air and Stream Improvement, Inc. March 2004.

[Bergman, 1995] Bergman, H. Tekniska högskolan in Lulea. Licentiate Essay 1995:14L. (In Swedish).

[FAO, 2005] FAOSTAT data (2005): http://faostat.fao.org/faostat/collections?subset=forestry, last accessed June 2005.

[Fischer et al., 2001] Fischer, Albrecht and Kämpfer. In: Kämpfer and Weissenfels. Biologische Abfälle. Springer Verlag. (In German).

[Ford-Robertson, 2003] Ford-Robertson, J.B. Implications of Harvested Wood Products Accounting - Analysis of issues raised by Parties to the UNFCCC and development of a Simple Decay approach. MAF Technical Paper No 2003/5, 30p. Ministry of Agriculture and Forestry, Wellington, New Zealand. http://www.maf.govt.nz/forestry/publications/index.htm.

[Gjesdal et al. , 1996] Gjesdal S.F.T., Flugsrud K., Mykkelbost T.C., and Rypdal K. A balance of use of wood products in Norway. Norwegian Pollution Control Authority SFT, Report 96:04, $54 \mathrm{p}$. 
[Hashimoto and Moriguchi , 2004] Hashimoto, S. and Moriguchi, Y. Data Book: Material and carbon flow of harvested wood in Japan. CGER-D034-2004. National Institute for Environmental Studies, Japan. Tsukuba. 40p. http://wwwcger.nies.go.jp/publication/D034/D034.pdf

[ Hyvönen et al. , 1998] Hyvönen, R., Ågren, G.I. and Bosatta , E. Predicting long-term soil carbon storage from short-term information. Soil Science Society of America Journal 62:1000-1005.

[IPCC, 1997] Intergovernmental Panel on Climate Change. Revised 1996 IPCC Guidelines for National Greenhouse Gas Inventories. IEA/OECD, Paris, 1997.

[IPCC, 2000] Intergovernmental Panel on Climate Change. Good Practice Guidance and Uncertainty Management in National Greenhouse Gas Inventories. Penman et al. (Ed.) IGES, Hayama, Japan, 2000.

[IPCC, 2003] Intergovernmental Panel on Climate Change. Good Practice Guidance for Land Use, Land-Use Change and Forestry. Penman et al. (Ed.) IGES, Hayama, Japan, 2003.

[Karjalainen et al. , 1994] Karjalainen, T., S. Kellomäki and A. Pussinen. Role of woodbased products in absorbing atmospheric carbon. Silva Fennica 28(2): 67-80, 1994.

[Marland and Marland, 2003] Marland, E. and G. Marland. The treatment of long-lived, carbon-containing products in inventories of carbon dioxide emissions to the atmosphere. Environmental Science and Policy, 6(2): 139-152, 2003.

[Pingoud et al. , 2001] Pingoud, K., Perälä, A.-L., Pussinen, A. Carbon dynamics in wood products. Mitigation and Adaptation Strategies for Global Change, Vol. 6, No. 2, pp. 91-111, 2001.

[Pingoud et al. , 1996] Pingoud K., Savolainen I., and Seppala H. Greenhouse impact of the Finnish forest sector including forest products and waste management. Ambio 25: 318-326.

[Row and Phelps, 1996] Row, C. and Phelps, R.B. Wood carbon flows and storage after timber harvest. In: Sampson, R.N., Hair, D. (Eds.), Forests and Global Change, vol. 2. Forest Management Opportunities for Mitigating Carbon Emissions. American Forests, Washington, DC, pp. 27-58. 